\title{
The diagnostic accuracy of truncated cardiovascular MR protocols for detecting non-ischemic cardiomyopathies
}

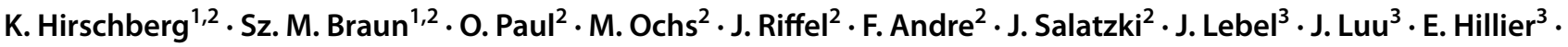 \\ M. Finster ${ }^{1} \cdot$ H. Vago ${ }^{1} \cdot$ B. Merkely ${ }^{1} \cdot$ H. A. Katus ${ }^{2} \cdot$ M. G. Friedrich ${ }^{2,3}$
}

Received: 2 September 2021 / Accepted: 27 October 2021 / Published online: 9 November 2021

(c) The Author(s) 2021

\begin{abstract}
Cardiovascular magnetic resonance imaging is one of the most important diagnostic modalities in the evaluation of cardiomyopathies. However, significant limitations are the complex and time-consuming workflows and the need of contrast agents. The aim of this multi-center retrospective study was to assess workflows and diagnostic value of a short, contrast agent-free cardiac magnetic resonance protocol. 160 patients from Heidelberg, Germany and 119 patients from Montreal, Canada with suspected cardiomyopathy and 20 healthy volunteers have been enrolled. Scans were performed at a 1.5Tesla or 3Tesla scanner in Heidelberg and at a 3Tesla scanner in Montreal. We used single-slice T1 map only. A stepwise analysis of images has been performed. The possible differential diagnosis after each step has been defined. T1-values and color-encoded T1 maps significantly contributed to the differential diagnosis in 54\% of the cases (161/299); the final diagnosis has been done without late gadolinium enhancement images in $83 \%$ of healthy individuals, in $99 \%$ of patients with dilated cardiomyopathy, in 93\% of amyloidosis patients, in $94 \%$ of patients with hypertrophic cardiomyopathy and in $85 \%$ of patients with hypertensive heart disease, respectively. Comparing the scan time with $(48 \pm 7 \mathrm{~min})$ vs. without contrast agent $(23 \pm 5 \mathrm{~min})$, significant time saving could be reached by the short protocol. Subgroup analysis showed the most additional diagnostic value of T1 maps in amyloidosis and hypertrophic cardiomyopathy or in confirmation of normal findings. In patients with unclear left ventricular hypertrophy, a short, non-contrast protocol can be used for diagnostic decision-making, if the quality of the T1 map is diagnostic, even if only one slice is available.
\end{abstract}

Keywords Cardiac imaging techniques $\cdot$ Magnetic resonance $\cdot$ Contrast agent $\cdot$ Cardiomyopathy $\cdot$ T1 mapping

\section{Introduction}

Cardiovascular magnetic resonance (CMR) imaging is the reference modality for assessing cardiac morphology and function, with a robust image quality, high accuracy, and excellent reproducibility [1,2]. Furthermore, CMR has a unique capability of myocardial tissue characterization, which is essential for identifying the presence, extent, severity, and etiology of myocardial injury in diseases such as

K. Hirschberg

hirschbergkristof@gmail.com

1 Heart and Vascular Center, Semmelweis University, Városmajor utca 68, Budapest 1122, Hungary

2 Department of Cardiology, Angiology and Pneumonology, University Hospital Heidelberg, Heidelberg, Germany

3 Departments of Medicine and Diagnostic Radiology, McGill University Health Centre, Montreal, Canada myocarditis, acute or chronic myocardial infarction, myocardial storage disease, cardiomyopathies [3-7]. Next to edema-sensitive CMR imaging using so-called T2-weighted sequences in the diagnostic workup of left ventricular dilatation, hypertrophy, or dysfunction, CMR can apply contrastenhanced imaging for identifying myocardial infiltration or scar. The most frequently used technique is called late gadolinium enhancement (LGE) imaging and allows for visualizing infiltration and irreversible injury (necrosis, scar). The observed regional distribution often helps establishing a definitive diagnosis with an impact on patient management $[8,9]$. In many clinical scenarios, the early implementation of CMR can save unnecessary diagnostic tests, speed-up decision making and reduce costs. As a significant limitation however, the current workflows for scanning and evaluation of CMR are complex and time-consuming, limiting its practical clinical utility (expensive hardware and software, operating costs, demanding training requirements 
for staff). Addressing some of these issues would lead to a wider availability for patients [10-16] and help improving informed therapeutic decision-making. Specifically, the need for injecting a contrast agent for tissue characterization in several clinical indications for CMR affects patient safety, comfort, and cost. Gadolinium-enhanced images prolong scan time significantly and require the availability of a physician in case of the rare event of an allergic reaction. Furthermore, some patients have contraindications to gadolinium such as severe kidney dysfunction or a known gadolinium allergy, or patients decline receiving contrast agents due to safety concerns $[17,18]$. For these reasons, contrast-agent free protocols, if without loss of relevant information, would be very beneficial.

Over the recent years, the techniques for myocardial T1and T2-mapping have led to the development of contrastagent free CMR protocols in patients with suspected cardiomyopathies [19-21]. The measurement of absolute values of global and segmental myocardial T1, displayed in colorencoded T1 maps is a viable alternative to conventional late gadolinium enhancement techniques in the detection of diffuse or focal fibrosis in different cardiac pathologies. Of note, conventional LGE imaging relies on a proper selection of MR sequence settings in order to "null" the signal from normal myocardium. In diffuse myocardial infiltration, especially at earlier stage, this may not be detected on late gadolinium enhanced images [19].

The aim of this multi-center retrospective study was to assess workflows and diagnostic value of a short, contrast agent-free CMR protocol in the evaluation of suspected cardiomyopathies when compared to standard CMR imaging. We aimed at evaluating both, global and regional myocardial abnormalities.

\section{Methods}

\section{Patients}

We performed a retrospective study conducted in the University Hospital Heidelberg, Germany and the McGill University Health Centre, Montréal, Canada. Both centers support large CMR clinical service (> 1000 patients a year). Approval for analysis of imaging data was granted by the local research ethics committees. We selected all patients with suspected cardiomyopathies who had undergone a complete CMR including cine images, T1 mapping and LGE (late gadolinium enhancement) between January 2016 and October $2017(n=299)$. The two cohorts consisted of 160 patients from Heidelberg and 119 patients from Montréal. In addition, 20 volunteers served as a control group for T1 mapping ( $n=10$ at $1.5 \mathrm{~T}$ and $\mathrm{n}=10$ at $3 \mathrm{~T})$. All of them were healthy with no significant medical history, no evidence of cardiovascular disease and without any regular medication. In both centers, all patients had provided written informed consent for the CMR examination including the administration of intravenous contrast agent. The patients' records were de-identified prior to analysis.

\section{CMR protocol}

The CMR procedures were performed by using a $1.5 \mathrm{~T}$ scanner (Philips Achieva 1.5T CX) and a 3T scanner (Philips Ingenia 3T) in Heidelberg and a 3T scanner (Siemens Magnetom Skyra 3T) in Montréal. 32-element cardiac receiver coils were used for all scanners. Subjects were scanned in supine position with vector ECG-gating. Short and long axis cine images covering the whole left ventricle were acquired using a multi-slice balanced steady state free precession (bSSFP) sequence. T1-mapping was performed using a 2D MOLLI 5s(3s)3s sequence in a single, mid-ventricular short axis slice during breath-hold in end-expiration at late diastole. This sequence is virtually independent from heart rate, but the recorded number of raw images is influenced by the heart rate and a minimum of 8 raw images were acquired with increasing inversion times (TI: $100-5000 \mathrm{~ms}$ ) at the papillary muscle level (flip angle: $35^{\circ}$ ) [22]. The reproducibility of T1-mapping has been evaluated during one of our previous studies, showing a small interobserver variability (mean difference of $8.2 \mathrm{~ms}$, and intraobserver $(5.9 \mathrm{~ms})$ variability at $1.5 \mathrm{~T}$ [23]. Late gadolinium enhancement (LGE) images were acquired starting 10 min after administration of Gadobutol (Gadovist, Schering, Berlin, Germany) $0.14 \mathrm{mmol} / \mathrm{kg}$ body weight $(1.5 \mathrm{~T})$ or $0.1 \mathrm{mmol} / \mathrm{kg}$ body weight (3T) employing a T1-weighted inversion recoveryprepared fast gradient echo sequence with an optimized inversion time. Regions with LGE were verified in at least one other orthogonal plane and in the same plane being obtained as a second image after changing the direction of readout [24].

\section{Image analysis}

All CMR scans were analysed using certified post-processing software (cvi42, version 5.2, Circle Cardiovascular Imaging Inc.) by an experienced cardiologist and certified CMR-specialist, who was blinded to the patients' diagnosis. Left endocardial and epicardial surfaces were contoured manually on the short axis cine images in order to obtain the morphological and functional indices: LV end systolic volume (LVESV), LV end diastolic volume (LVEDV), LV septal thickness, LV ejection fraction (LVEF) calculated from the end systolic and end diastolic volumes. For LV mass calculation, intraventricular septum was included. CMR volume and mass measurements were indexed for body surface area where appropriate. Native T1 maps were generated and 
analysed by using the same software package. All maps were created by a trained CMR researcher blinded to the original diagnosis and previous results. An R2 cut-off value of 0.9 was used to avoid the generation of false pixels resulting from some form of curve fitting errors. For contouring an offset of $10 \%$ was used to avoid partial-volume effects in the subendocardial and subepicardial layers. Global T1 values were calculated as a mean of the 6 segments of the single, mid-ventricular short axis slice. Polar maps were created based on the mid-ventricular short axis slice of the American Heart Association (AHA) 17-segment model [25]. By using our normal reference values calculated from the healthy volunteers, we created specific color-encoded T1 maps to facilitate the visualisation of any pathologic changes. We used green color to highlight areas within normal range of $\mathrm{T} 1$ relaxation times, yellow, blue and red colors for increased, decreased T1 values and for blood pool, respectively. Myocardial $\mathrm{T} 1$ were considered abnormal if the mean value or at least 10 contiguous pixels $(\sim 4 \mathrm{~mm})$ had a T1 value out of the normal range. LGE images were visually analysed for the presence or absence of enhancement.

\section{Three-step blinded analysis of images}

All data and images were analysed in three steps to get to a final diagnosis. After each step, the possible number of differential diagnoses were considered. At the first step, only the patient`s history and morphology (cine images) were evaluated. It was followed by the evaluation of the T1 maps (second step). Finally, LGE images were used to further reduce the number of possible differential diagnoses or to verify the suspected diagnosis (third step). After the diagnostic procedure, all patients were assigned to the following groups according to their final diagnosis: normal findings, amyloidosis, DCM (dilated cardiomyopathy), HCM (hypertrophic cardiomyopathy), HHD (hypertensive heart disease), unclear diagnosis, other diagnosis. If no diagnosis could be stated after the third step, no single final diagnosis was given. "Other diagnosis" was stated if any other diagnosis except the above mentioned (normal or amyloidosis or DCM or HCM or HHD) was found.

\section{Statistical analysis}

Categorical data are expressed as percentages and continuous variables as mean \pm SD. Statistical significance was defined for $p$ values $<0.05$. Groups were compared by using unpaired Student's t-test. All tests were 2-tailed. Normal reference range for $\mathrm{T} 1$ values was calculated from the results of healthy volunteers, defined as mean $\pm 1.96 \mathrm{SD}$ as previously suggested [19].

\section{Results}

The normal ranges for $1.5 \mathrm{~T}$ and $3 \mathrm{~T}$ were $956-1035 \mathrm{~ms}$ and 1197-1286 ms, respectively. From the Heidelberg cohort $(n=160), 14$ patients were excluded from the final analysis because of an unclear diagnosis or specific ischemic changes or other diagnosis. These patients were assigned to the following groups: Normal, $\mathrm{n}=16$; amyloidosis, $\mathrm{n}=23$; DCM, $\mathrm{n}=56$; HCM, $\mathrm{n}=30$; HHD, $\mathrm{n}=21$. From the Montreal cohort $(n=119), 31$ patients were excluded because of an unclear diagnosis or other specific diagnoses. These patients were assigned to the following groups: normal, $\mathrm{n}=25$; amyloidosis, $\mathrm{n}=4$; $\mathrm{DCM}, \mathrm{n}=30$; HCM, $\mathrm{n}=17$; HHD, $\mathrm{n}=12$. After the exclusion of 45 patients, a total of 234 patients were finally analysed.

\section{Scan time}

We analysed the time needed for the CMR examinations. The mean scan time when including post-contrast images was $48 \pm 7 \mathrm{~min}$, while images without using contrast-enhanced sequences were acquired in $23 \pm 5 \mathrm{~min}$; $\mathrm{p}<0.0001$.

\section{Clinical characteristics of patients and CMR results}

Baseline clinical characteristics and CMR findings of the healthy volunteers and patients are summarised in Tables 1,2 and 3. All results of the patients were compared to those of the volunteers.

\section{Patients with normal findings}

In both cohorts, $\mathrm{T} 1$ values did not differ from that of the healthy volunteers $(995.6 \mathrm{~ms}$ at $1.5 \mathrm{~T}$ and $1241.1 \mathrm{~ms}$ at $3 \mathrm{~T}$ ), confirming the absence of pathological alterations of the myocardium.

Figure $1 \mathrm{M}-\mathrm{O}$ shows a representative color-encoded $\mathrm{T} 1$ map in a healthy subject.

\section{Amyloidosis}

As expected, LV mass indices and septal thickness showed a robust increase in both cohorts $\left(93.2 \mathrm{~g} / \mathrm{m}^{2}\right.$ and $131.8 \mathrm{~g} /$ $\left.\mathrm{m}^{2}\right)$ when compared to the healthy volunteers $\left(48.6 \mathrm{~g} / \mathrm{m}^{2}\right.$, $\mathrm{p}<0.05)$. T1 was also significantly increased; $1164 \mathrm{~ms}$ at $1.5 \mathrm{~T}$ and $1407 \mathrm{~ms}$ and $1439 \mathrm{~ms}$ at 3T (Tables 2 and 3, Fig. 1A-C). 
Table 1 Clinical and CMR characteristics of healthy volunteers

\begin{tabular}{lll}
\hline & Healthy volunteers, $\mathrm{n}=20$ \\
\hline Clinical characteristics & & \\
Age (years) & $58.9 \pm 11.2$ & \\
Gender, $\mathrm{n}($ female/male) & $6 / 14$ & \\
BMI $\left(\mathrm{kg} / \mathrm{m}^{2}\right)$ & $23.6 \pm 2.6$ & \\
hsTnT $(\mathrm{pg} / \mathrm{l})$ & $5.8(3-10), \mathrm{n}=6$ & \\
NT-proBNP $(\mu \mathrm{g} / \mathrm{ml})$ & $87.8(20-179), \mathrm{n}=6$ & \\
GFR $\left(\mathrm{ml} / \mathrm{min} / 1.73 \mathrm{~m}^{2}\right.$ [CKD-EPI]) & $89.0 \pm 11.4$ & \\
Creatinine $(\mu \mathrm{mol} / \mathrm{l})$ & $86.0 \pm 11.8$ & \\
Hypertension, $\mathrm{n}$ & 0 & \\
Diabetes mellitus, $\mathrm{n}$ & 0 & $\mathrm{n}=10$ \\
Hyperlipidaemia, $\mathrm{n}$ & 0 & \\
Smoking history, $\mathrm{n}$ & 0 & \\
Familiy history of CVD & 0 & \\
CMR characteristics & & \\
LVEDV (ml $\left./ \mathrm{m}^{2}\right)$ & $84.5 \pm 13.0$ & \\
LVESV (ml/m ${ }^{2}$ ) & $32.6 \pm 6.1$ & \\
LVEF $(\%)$ & $61.5 \pm 3.3$ & \\
LV mass $\left(\mathrm{g} / \mathrm{m}^{2}\right.$ ) & $48.6 \pm 8.2$ & \\
Septal thickness (mm) & $9.2 \pm 1.9$ & \\
Presence of LGE/total, $\mathrm{n}$ & $0 / 20$ & \\
Native T1 value at $1.5 T(\mathrm{~ms})$ & $995.6 \pm 20.2$ & \\
Native T1 value at 3T $(\mathrm{ms})$ & $1241.1 \pm 22.7$ & \\
\hline
\end{tabular}

Values are expressed as mean \pm SD. Number of patients are presented where it differed from the patient group

BMI body mass index, hsTnT high-sensitivity Troponin T, NTproBPN N-terminal pro b-type natriuretic peptide, GFR glomerular filtration rate, LVEDV left ventricular end-diastolic volume, LVESV left ventricular end-systolic volume, LV left ventricular, LGE late gadolinium enchancement

\section{Dilated cardiomyopathy}

LVEDV $\left(119.9 \mathrm{ml} / \mathrm{m}^{2}\right.$ and $\left.132.8 \mathrm{ml} / \mathrm{m}^{2}\right)$, LVESV $(74.0 \mathrm{ml} /$ $\mathrm{m}^{2}$ and $\left.92.3 \mathrm{ml} / \mathrm{m}^{2}\right)$, LV mass indices $\left(69.5 \mathrm{~g} / \mathrm{m}^{2}\right.$ and $\left.103.7 \mathrm{~g} / \mathrm{m}^{2}\right)$ were significantly higher and LVEF $(40.3 \%$ and $31.2 \%$ ) was significantly lower in both cohorts as compared to the healthy volunteers (LVEDV $84.5 \mathrm{ml} / \mathrm{m}^{2}$, LVESV $32.6 \mathrm{ml} / \mathrm{m}^{2}$, LV mass $48.6 \mathrm{~g} / \mathrm{m}^{2}$, LVEF $61.5 \%$, $\mathrm{p}<0.05$, respectively).

T1 values were slightly, but significantly elevated; $1025.6 \mathrm{~ms}$ at $1.5 \mathrm{~T}$ and $1274.6 \mathrm{~ms}$ and $1306.1 \mathrm{~ms}$ at $3 \mathrm{~T}$ (Tables 2 and 3, Fig. 1D-F).

\section{Hypertrophic cardiomyopathy}

The difference between the T1 values was not as prominent as in amyloidosis, albeit still significantly higher than normal; $1027.4 \mathrm{~ms}$ at $1.5 \mathrm{~T}$ and $1270.3 \mathrm{~ms}$ and $1292.2 \mathrm{~ms}$ at $3 \mathrm{~T}$ vs. $995.6 \mathrm{~ms}$ at $1.5 \mathrm{~T}$ and $1241.1 \mathrm{~ms}$ at $3 \mathrm{~T}$, respectively $(\mathrm{p}<0.05)$. The most prominent differences in both groups were in the LV mass indices $\left(78.0 \mathrm{~g} / \mathrm{m}^{2}\right.$ and $103.1 \mathrm{~g} /$ $\mathrm{m}^{2}$ vs. $\left.48.6 \mathrm{~g} / \mathrm{m}^{2}, \mathrm{p}<0.05\right)$ and septal thickness $(17.8 \mathrm{~mm}$ and $13.6 \mathrm{~mm}$ vs. $9.2 \mathrm{~mm}, \mathrm{p}<0.05)$. LVESV decreased significantly (Tables 2 and 3, Fig. 1G-I).

\section{Hypertensive heart disease}

There were 33 patients diagnosed with HHD in the two cohorts together. BMI was significantly higher in both cohorts $\left(31.6 \mathrm{~kg} / \mathrm{m}^{2}\right.$ and $29.4 \mathrm{~kg} / \mathrm{m}^{2}$ vs. $23.6 \mathrm{~kg} / \mathrm{m}^{2}$, $\mathrm{p}<0.05)$. Both LV mass indices and septal thickness were significantly higher in both the Heidelberg and Montreal cohorts $\left(70.2 \mathrm{~g} / \mathrm{m}^{2}\right.$ and $88.7 \mathrm{~g} / \mathrm{m}^{2}$ vs. $48.6 \mathrm{~g} / \mathrm{m}^{2} ; 12.9 \mathrm{~mm}$ and $11.2 \mathrm{~mm}$ vs. $9.2 \mathrm{~mm}$, respectively, p<0.05). In the Heidelberg cohort, there was no significant difference between the T1 values ( $1248 \mathrm{~ms}$ vs. $1239 \mathrm{~ms}$ at $3 \mathrm{~T}$ and $1010 \mathrm{~ms}$ vs. $1011 \mathrm{~ms}$ at $1.5 \mathrm{~T}$, both $\mathrm{p}>0.4)$. In the Montreal cohort, $\mathrm{T} 1$ values were significantly higher $(1301 \mathrm{~ms} ; \mathrm{p}<0.05)$. (Tables 2 and 3, Fig. 1J-L).

\section{T1-mapping in the stepwise diagnostic approach}

The evaluation of the patients' data and images was performed in three steps by an experienced cardiologist, blinded to the diagnoses as stated on the clinical CMR report. Table 4 shows the reduction of differential diagnosis under consideration when adding T1 mapping to the analysis.

\section{Step 1}

After checking the patient's history and completing the morphological analysis of 234 patients, in 94 cases (40\%) a final diagnosis could be made (74\% of DCM cases, $34 \%$ of HCM cases, but none of the amyloidosis cases could be diagnosed at this step).

\section{Step 2}

In this step, polar maps and color-coded $\mathrm{T} 1$ maps were added to the cine images. In additional 122 cases a diagnosis could be established ( 216 from 234 cases, $92 \%$ in total). The greatest benefit of T1-mapping was seen during the evaluation of patients with amyloidosis, followed by HCM. In amyloidosis patients, a globally high native myocardial $\mathrm{T} 1$ reliably visualized diffuse myocardial infiltration. T1-mapping was sufficient for the diagnosis in 25 cases, representing 93\% of all amyloidosis cases (Table 4). In HCM patients, focal fibrosis at the RV insertion points was often well visualised on native color-coded T1 maps. T1-mapping helped to diagnose 28 additional cases of $\mathrm{HCM}(60 \%$ of all $\mathrm{HCM})$ and 22 more cases of HHD (67\% of all HHD). Diagnostic step 1 and 2 together made it possible to diagnose 44 cases of HCM (94\% of all HCM) and 28 cases of HHD (85\% of 


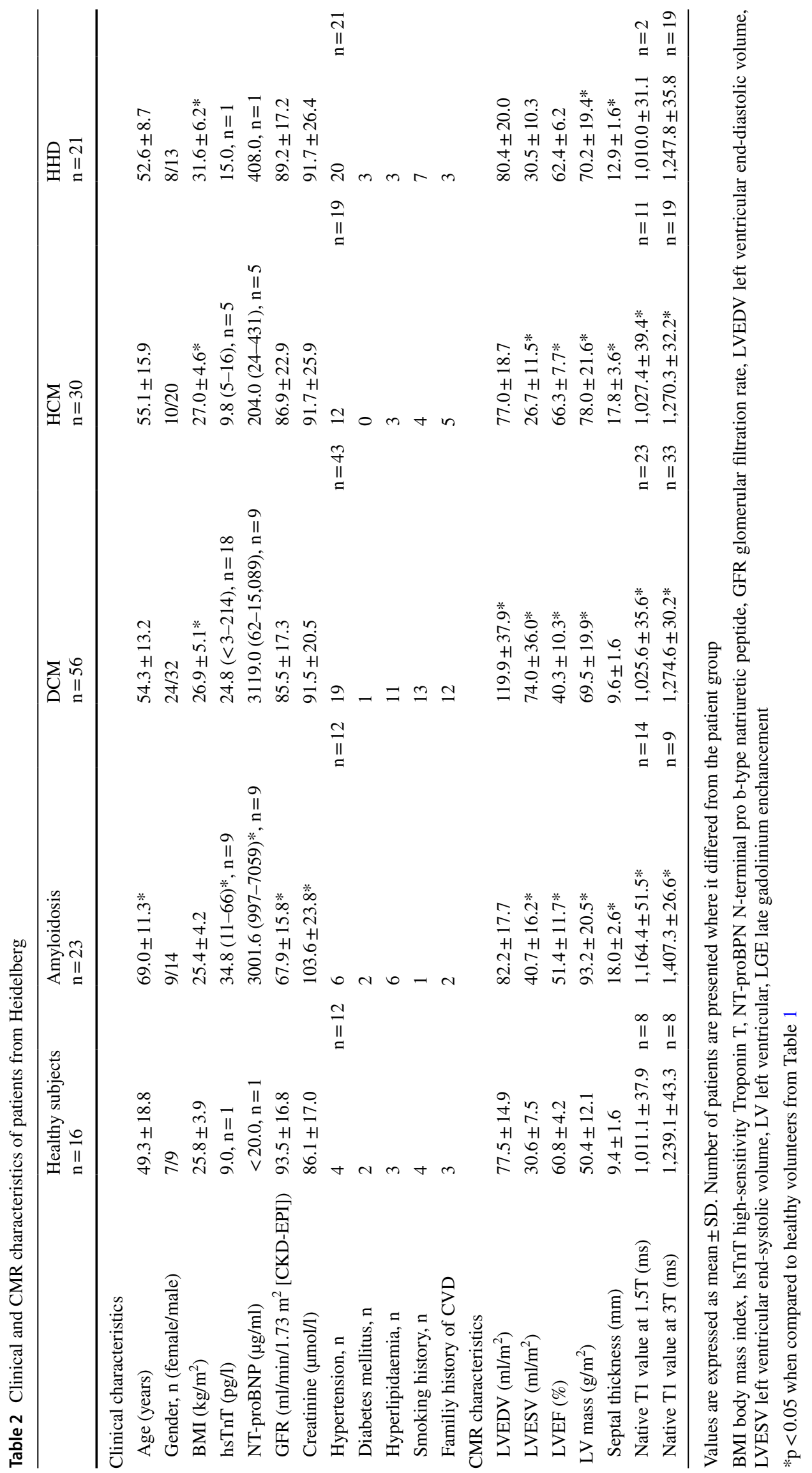




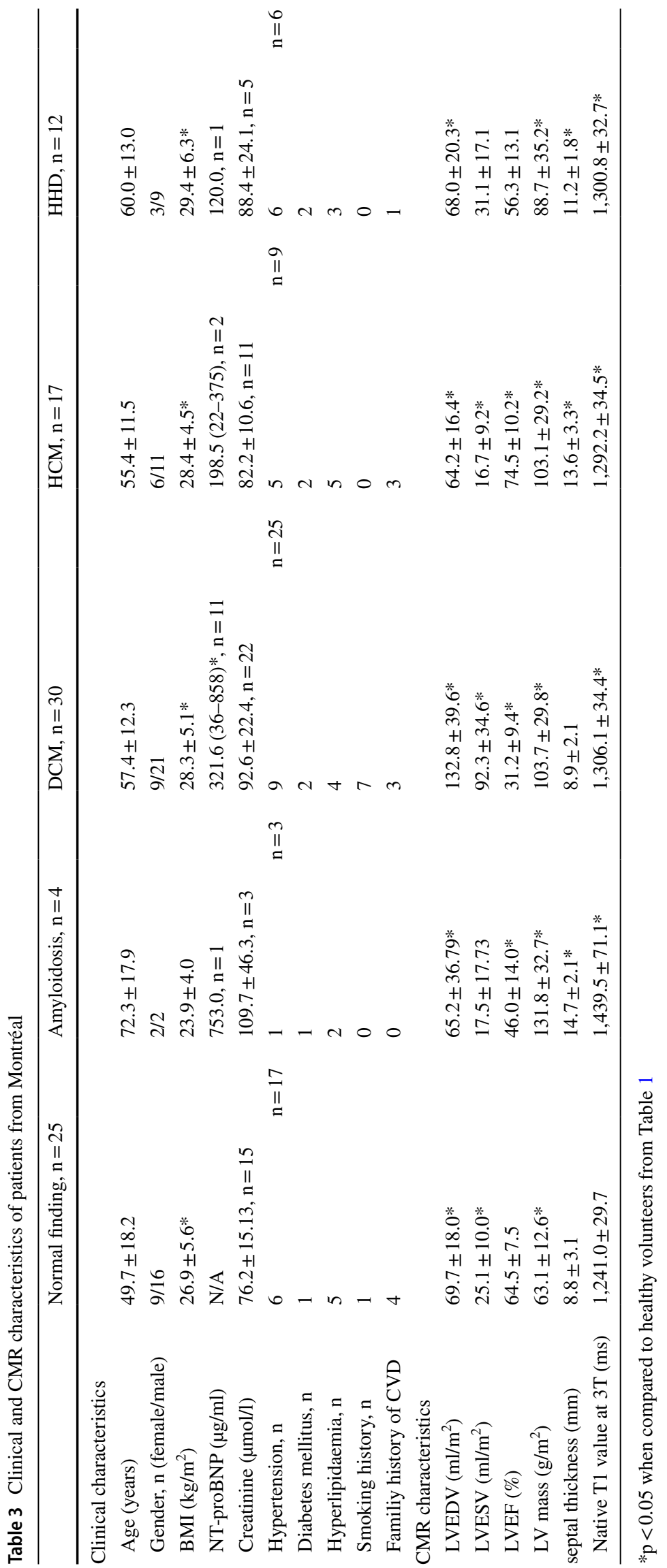




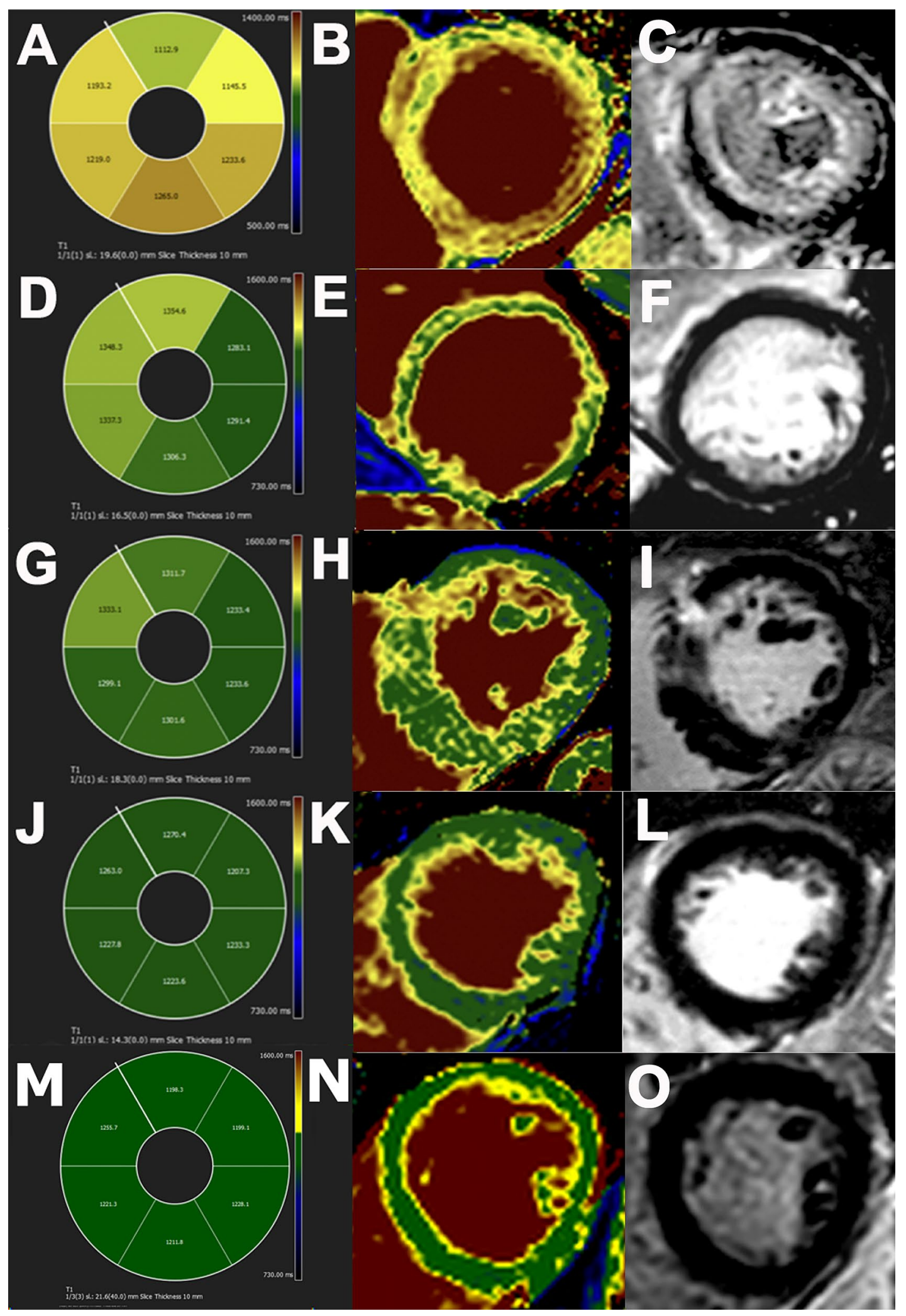

Fig. 1 A-C T1 polar map, color-coded T1 map and the correspondent LGE image of a patient with amyloidosis. D-F T1 polar map, color-coded T1 map and the correspondent LGE image of a patient with DCM. G-I T1 polar map, color-coded T1 map and the cor- respondent LGE image of a patient with HCM. J-L T1 polar map, color-coded T1 map and the correspondent LGE image of a patient with hypertensive heart disease (HHD). M-O T1 polar map, colorcoded T1 map and the correspondent LGE image of healthy volunteer 
all HHD). Looking at the subgroup of patients with normal finding, T1-mapping ruled out the possibility of myocardial disease in 26 more cases (63\% of all individuals in this group), which means 34 cases together with the first step ( $83 \%$ of all individuals in this group).

\section{Step 3}

The final step of the evaluation included the additional analysis of LGE images. There were 31 cases, where neither LGE nor the previous methods were able to reduce the possible number of differential diagnoses to a single one. Just like the patients with "other diagnoses" $(n=14)$, these patients were also excluded from the final interpretation. For the correct final diagnosis, LGE was essential in 18 cases (8\%). Figure 2 shows the stepwise narrowing of differential diagnoses (DDx) after each step.

Representative examples of color-encoded T1 maps from a healthy volunteer and from each subgroup are shown in Fig. 1. The incremental diagnostic yield is shown in Fig. 3.

Table 4 Results of the three-step evaluation

\begin{tabular}{lllll}
\hline Final diagnosis & $\begin{array}{l}\text { Total number of } \\
\text { patients }\end{array}$ & $\begin{array}{l}\text { Correct diagnosis (his- } \\
\text { tory+morphology) }\end{array}$ & $\begin{array}{l}\text { Correct diagnosis (history + mor- } \\
\text { phology+ T1-mapping) }\end{array}$ & $\begin{array}{l}\text { Correct diagnosis (his- } \\
\text { tory + morphology + T1-map- } \\
\text { ping + LGE) }\end{array}$ \\
\hline Healthy subjects & 41 & $8(19.5 \%)$ & $34(83 \%)$ & $41(100 \%)$ \\
Amyloidosis & 27 & $0(0 \%)$ & $25(93 \%)$ & $27(100 \%)$ \\
DCM & 86 & $64(74 \%)$ & $85(99 \%)$ & $86(100 \%)$ \\
HCM & 47 & $16(34 \%)$ & $44(94 \%)$ & $47(100 \%)$ \\
HHD & 33 & $6(18 \%)$ & $28(85 \%)$ & $33(100 \%)$ \\
\hline
\end{tabular}

The percentage of hitting the final diagnosis made by the inclusion of LGE images is shown at each diagnostic steps

Values show the total number of correct diagnoses made after each step

LGE late gadolinium enhancement, DCM dilated cardiomyopathy, HCM hypertrophic cardiomyopathy, HHD hypertensive heart disease

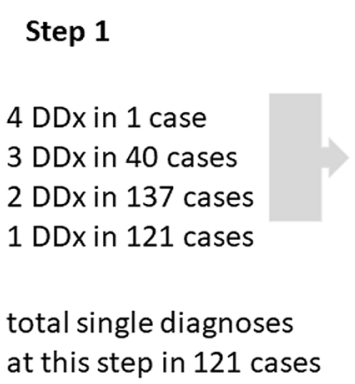

Step 2

3 DDx in 5 cases
2 DDx in 49 cases
1 DDx in 124 cases

total single diagnoses

at this step in 245 cases
Step 3

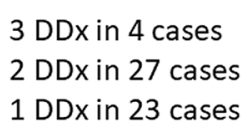

total single diagnoses at this step in 268 cases

Fig. 2 Narrowing of differential diagnoses (DDx) at each step

Fig. 3 Incremental diagnostic yield in healthy individuals and in DCM, amyloidosis, HCM and HHD patients
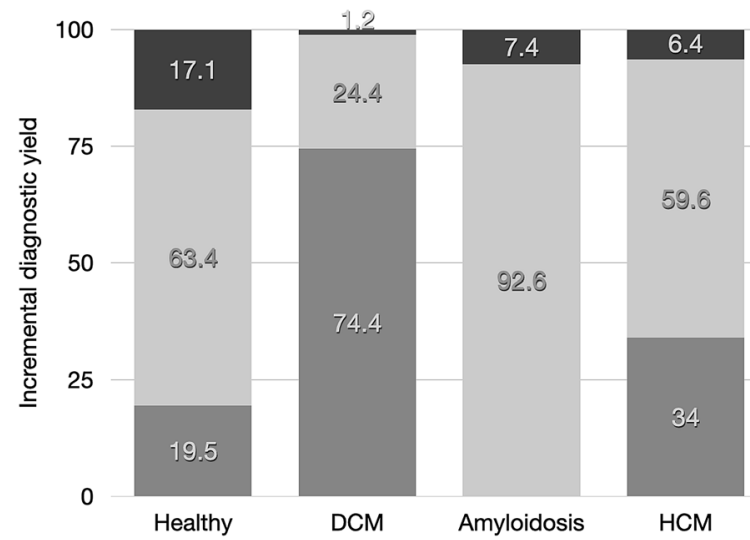

15.1

31 cases remained still unclear after step 3 


\section{Discussion}

Our study in two experienced CMR centres showed that in $92 \%$ of patients with suspected non-ischemic cardiomyopathies, scan time and associated costs could be significantly reduced by omitting contrast-enhanced images without impairment of diagnostic performance. In the remaining $8 \%$ only, readers required contrast-enhanced LGE images were necessary for diagnostic decision-making. The results however vary between cardiomyopathies. While the performance of non-contrast enhanced protocols was excellent in DCM (99\%), in HCM (94\%) and amyloidosis $(93 \%)$, the accuracy was below $90 \%$ in patients with hypertensive heart disease. In $83 \%$ of normal individuals, readers felt comfortable ruling out cardiac disease without LGE images.

Our findings have several clinical implications: Contrast agents may be omitted in HCM and DCM, the two most frequent cardiomyopathies. The results do also provide reassurance that in patients with suspected cardiomyopathies in whom the injection of gadolinium is contraindicated, a CMR with a high diagnostic yield can be performed even without the use of a contrast agent.

Our results also support a non-rigid protocol, i.e. advancing to contrast-enhanced images only in the few cases where the diagnosis cannot be established based on cine and mapping alone. Such a strategy would save costs, albeit would require a knowledge-based decision during the scan. One could even consider offering patients a shortened, non-contrast protocol and add the contrastenhanced part at a second scan, if necessary.

T1 maps with diagnostic quality are an essential component in this approach. The quality and utility of T1 mapping requires suitable hardware and software, as well as expertise and experience of the readers. Furthermore, the quality of T1 maps may vary, and the accuracy also relies on local quality standards such as center-specific normal values and validation in phantoms. As for LGE imaging, T1 mapping should ideally be applied as a stack as an incomplete coverage of the LV reduces its sensitivity for focal lesions (e.g. apical type of hypertrophic cardiomyopathy). In addition, while the global T1 values in our healthy subjects were all within these reference ranges, in some few cases, mild, clinically not relevant, focal changes might have occurred [26].

We observed a very consistent, strong T1 increase in amyloidosis, confirming the high diagnostic accuracy of T1-mapping in cardiac amyloidosis [6, 19, 27]. Considering the difficulties of LGE imaging in these patients [28], our results support CMR protocols without contrast agents in these patients.

Our findings of a globally increased T1 in DCM are consistent with previous reports [29], including its co-location with LGE [30, 31]. The diagnosis of DCM is based on the presence of reduced myocardial function and volume dilatation in the absence of ischaemic heart disease [32]. The importance of morphological and functional analysis can be clearly seen in our three-step diagnostic protocol, where $74 \%$ of DCM patients have been diagnosed based on morphology alone and $99 \%$ when adding mapping. In clinical scenarios, it can be challenging to diagnose the disease at early stages. For such cases, T1-mapping may be very useful especially for early antifibrotic medication [32].

In patients with HCM, we found significantly higher myocardial $\mathrm{T} 1$ values when compared to healthy volunteers. The focal elevation of $\mathrm{T} 1$ times, mostly at the insertion zones of the right ventricle, were corresponding with abnormal LGE in these regions [33]. Furthermore, in 15\% of HCM cases, T1-mapping revealed more focal lesions than did contrastenhanced LGE imaging. This observation is consistent with previous reports on an additional value of T1 mapping beyond LGE imaging [34]. We encountered 3 cases with negative $\mathrm{T} 1$ maps, likely because the incomplete coverage of the left ventricle by just one mid-ventricular slice. It is known, that $\mathrm{T} 1$ may very between different regions of the left ventricle, a strong argument for better coverage by more slices [35].

In arterial hypertension, myocardium remodeling occurs due to cardiomyocyte hypertrophy, fibroblast stimulation and increased collagen deposition. Both, left ventricular hypertrophy and the accumulation of interstitial collagen fibers are progressive [36]. The progressivity of remodeling can be the explanation to the difference of $\mathrm{T} 1$ values in our patient cohorts. Patients from Montréal showed a distinct T1 time elongation, while patients from Heidelberg had only a slightly increased mean $\mathrm{T} 1$ value, which suggests that the patients from Heidelberg were at an early stage of the disease. In the HHD subgroup, $76 \%$ of patients showed a positive T1 map in contrast with the $33 \%$ with LGE imaging. Although T1 mapping patterns are not always diseasespecific, T1-mapping has a clear advantage in revealing early fibrotic transformation.

In our study, a major contribution of T1-mapping was the addition of diagnostic information in patients with left ventricular hypertrophy of unknown origin. The absolute value of $\mathrm{T} 1$ and its regional distribution pattern help differentiate between HCM, amyloidosis and hypertensive heart disease $[19,37,38]$.

Nadjiri et al. recently evaluated a shortened protocol in a clinical setting in 160 patients [39]. The authors investigated a more heterogenous patient collective compared to our patients, since we focused only on the evaluation of suspected cardiomyopathies and acute cardiac pathologies such as myocardial infarction or myocarditis has been excluded. Similar to our results, Nadjiri et al. showed that a shortened protocol 
comprised only of myocardial T1 mapping and cine images can discriminate patients who will benefit from a full contrast CMR protocol from those who do not.

T1 mapping, especially when showing an increase of myocardial T1, covers a wider range of myocardial pathology as compared to T2 mapping [21]. T2 on the other hand can specifically identify acute myocardial injury through visualizing myocardial edema. We omitted T2 mapping in our study because in the setting of chronic myocardial disease, T1 mapping has a broader range of detectable myocardial pathologies.

In summary, the following pragmatic approach for a shortened, contrast agent-free CMR protocol could be clinically applied in patients with suspected cardiomyopathy: (1) cine images and $\mathrm{T} 1$ mapping are acquired and immediately analyzed (while patient is in the scanner). A normal myocardial T1 and normal ventricular morphology and function as assessed in a set of diagnostic T1 maps and cine images allow for ruling out cardiomyopathy. (2) In case of LV hypertrophy and typical T1 abnormalities indicating HCM or amyloidosis, no LGE images and thus no contrast application would be necessary to rule in HCM or amyloidosis. In all other cases, the scan could be expanded to a full protocol with contrast-enhanced images or a focused contrast-enhanced CMR scan could be scheduled.

\section{Limitations}

There are several, very important limitations of this study. Besides its retrospective design, our study may have been affected by significant selection bias, since only patients with suspected CMP and a clear final diagnosis of a nonischemic CMP were included. We excluded acute clinical syndromes, ischemic pathologies, and rare diagnoses such as arrhythmogenic cardiomyopathy, cardiac involvement of systemic diseases, sarcoidosis etc. Therefore, our results are not applicable to acute myocardial injury and infrequent cardiomyopathies. We believe would however expect similarly positive results. Furthermore, sensitivity and specificity and AUC could not be calculated from these cohorts. We only analyzed one midventricular short axis T1 map, and T2 maps were not included. Thus, updated protocols as now used in many centres would likely achieve even better results. It is also important to state, that omitting LGE imaging may forfeit the opportunity to acquire additional prognostic information.

\section{Conclusions}

Our results indicate that CMR using a truncated protocol of combined functional (cine) images and a single-slice T1 map may in most of patients be sufficient to rule out or confirm the diagnosis of several non-ischemic cardiomyopathies, specifically hypertrophic and dilated forms. These protocol allow for omitting the administration of contrast agents and could significantly reduce the duration and cost of CMR exams. These findings should be confirmed with updated CMR protocols using multi-slice T1 and T2 maps, and in larger, prospective studies.

Funding Open access funding provided by Semmelweis University. The research was financed by the Thematic Excellence Programme (2020-4.1.1.-TKP2020) of the Ministry for Innovation and Technology in Hungary, within the framework of the Therapeutic Development and Bioimaging thematic programmes of the Semmelweis University. Project no. NVKP_16-1-2016-0017 ('National Heart Program') has been implemented with the support provided from the National Research, Development and Innovation Fund of Hungary, financed under the NVKP_16 funding scheme.

Availability of data and material On request from the corresponding author.

Code availability Not applicable.

\section{Declarations}

Conflict of interest Matthias G. Friedrich is board member, shareholder, and consultant of Circle Cardiovascular Imaging Inc.

Ethics approval McGill University and Heidelberg University.

Consent to participate Not applicable.

Consent for publication Not applicable.

Open Access This article is licensed under a Creative Commons Attribution 4.0 International License, which permits use, sharing, adaptation, distribution and reproduction in any medium or format, as long as you give appropriate credit to the original author(s) and the source, provide a link to the Creative Commons licence, and indicate if changes were made. The images or other third party material in this article are included in the article's Creative Commons licence, unless indicated otherwise in a credit line to the material. If material is not included in the article's Creative Commons licence and your intended use is not permitted by statutory regulation or exceeds the permitted use, you will need to obtain permission directly from the copyright holder. To view a copy of this licence, visit http://creativecommons.org/licenses/by/4.0/.

\section{References}

1. Kinno M, Nagpal P, Horgan S, Waller AH (2017) Comparison of echocardiography, cardiac magnetic resonance, and computed tomographic imaging for the evaluation of left ventricular myocardial function: part 1 (global assessment). Curr Cardiol Rep 19(1):9

2. Li C, Lossnitzer D, Katus HA, Buss SJ (2012) Comparison of left ventricular volumes and ejection fraction by monoplane cineventriculography, unenhanced echocardiography and 
cardiac magnetic resonance imaging. Int J Cardiovasc Imaging 28(5):1003-1010

3. Authors/Task Force m, Elliott PM, Anastasakis A, Borger MA, Borggrefe M, Cecchi F et al (2014) 2014 ESC Guidelines on diagnosis and management of hypertrophic cardiomyopathy: the Task Force for the Diagnosis and Management of Hypertrophic Cardiomyopathy of the European Society of Cardiology (ESC). Eur Heart J 35(39):2733-2779

4. Dastidar AG, Harries I, Pontecorboli G, Bruno VD, De Garate E, Moret C et al (2019) Native T1 mapping to detect extent of acute and chronic myocardial infarction: comparison with late gadolinium enhancement technique. Int $\mathbf{J}$ Cardiovasc Imaging 35(3):517-527

5. Friedrich MG, Sechtem U, Schulz-Menger J, Holmvang G, Alakija P, Cooper LT et al (2009) Cardiovascular magnetic resonance in myocarditis: a JACC White Paper. J Am Coll Cardiol 53(17):1475-1487

6. Karamitsos TD, Piechnik SK, Banypersad SM, Fontana M, Ntusi NB, Ferreira VM et al (2013) Noncontrast T1 mapping for the diagnosis of cardiac amyloidosis. JACC Cardiovasc Imaging 6(4):488-497

7. Ruberg FL (2013) T1 mapping in cardiac amyloidosis: can we get there from here? JACC Cardiovasc Imaging 6(4):498-500

8. Bruder O, Wagner A, Lombardi M, Schwitter J, van Rossum A, Pilz G et al (2013) European Cardiovascular Magnetic Resonance (EuroCMR) registry-multi national results from 57 centers in 15 countries. J Cardiovasc Magn Reson 15:9

9. Ponikowski P, Voors AA, Anker SD, Bueno H, Cleland JGF, Coats AJS et al (2016) 2016 ESC Guidelines for the diagnosis and treatment of acute and chronic heart failure: The Task Force for the diagnosis and treatment of acute and chronic heart failure of the European Society of Cardiology (ESC) Developed with the special contribution of the Heart Failure Association (HFA) of the ESC. Eur Heart J 37(27):2129-2200

10. Kido T, Kido T, Nakamura M, Watanabe K, Schmidt M, Forman $\mathrm{C}$ et al (2016) Compressed sensing real-time cine cardiovascular magnetic resonance: accurate assessment of left ventricular function in a single-breath-hold. J Cardiovasc Magn Reson 18(1):50

11. Kramer CM, Barkhausen J, Bucciarelli-Ducci C, Flamm SD, Kim RJ, Nagel E (2020) Standardized cardiovascular magnetic resonance imaging (CMR) protocols: 2020 update. J Cardiovasc Magn Reson 22(1): 17

12. Kramer CM, Barkhausen J, Flamm SD, Kim RJ, Nagel E (2008) Society for Cardiovascular Magnetic Resonance Board of Trustees Task Force on Standardized P. Standardized cardiovascular magnetic resonance imaging (CMR) protocols, society for cardiovascular magnetic resonance: board of trustees task force on standardiz. J Cardiovasc Magn Reson 10:35

13. Kramer CM, Barkhausen J, Flamm SD, Kim RJ, Nagel E (2013) Society for Cardiovascular Magnetic Resonance Board of Trustees Task Force on Standardized P. Standardized cardiovascular magnetic resonance (CMR) protocols 2013 update. J Cardiovasc Magn Reson 15:91

14. McKenna WJ, Maron BJ, Thiene G (2017) Classification, epidemiology, and global burden of cardiomyopathies. Circ Res 121(7):722-730

15. Plein S, Schulz-Menger J, Almeida A, Mahrholdt H, Rademakers F, Pennell D et al (2011) Training and accreditation in cardiovascular magnetic resonance in Europe: a position statement of the working group on cardiovascular magnetic resonance of the European Society of Cardiology. Eur Heart J 32(7):793-798

16. Timmis A, Townsend N, Gale C, Grobbee R, Maniadakis N, Flather M et al (2018) European Society of Cardiology: Cardiovascular Disease Statistics 2017. Eur Heart J 39(7):508-579
17. Radbruch A (2018) Gadolinium deposition in the brain: we need to differentiate between Chelated and Dechelated gadolinium. Radiology 288(2):434-435

18. McDonald RJ, McDonald JS, Kallmes DF, Jentoft ME, Murray DL, Thielen KR et al (2015) Intracranial Gadolinium deposition after contrast-enhanced MR imaging. Radiology 275(3):772-782

19. Messroghli DR, Moon JC, Ferreira VM, Grosse-Wortmann L, He $\mathrm{T}$, Kellman P et al (2017) Clinical recommendations for cardiovascular magnetic resonance mapping of $\mathrm{T} 1, \mathrm{~T} 2, \mathrm{~T} 2 *$ and extracellular volume: a consensus statement by the Society for Cardiovascular Magnetic Resonance (SCMR) endorsed by the European Association for Cardiovascular Imaging (EACVI). J Cardiovasc Magn Reson 19(1):75

20. Messroghli DR, Moon JC, Ferreira VM, Grosse-Wortmann L, He T, Kellman P et al (2018) Correction to: Clinical recommendations for cardiovascular magnetic resonance mapping of T1, T2, $\mathrm{T} 2 *$ and extracellular volume: A consensus statement by the Society for Cardiovascular Magnetic Resonance (SCMR) endorsed by the European Association for Cardiovascular Imaging (EACVI). J Cardiovasc Magn Reson 20(1):9

21. Moon JC, Messroghli DR, Kellman P, Piechnik SK, Robson MD, Ugander M et al (2013) Myocardial T1 mapping and extracellular volume quantification: a Society for Cardiovascular Magnetic Resonance (SCMR) and CMR Working Group of the European Society of Cardiology consensus statement. J Cardiovasc Magn Reson 15:92

22. Messroghli DR, Greiser A, Frohlich M, Dietz R, Schulz-Menger J (2007) Optimization and validation of a fully-integrated pulse sequence for modified look-locker inversion-recovery (MOLLI) T1 mapping of the heart. J Magn Reson Imaging 26(4):1081-1086

23. aus dem Siepen F, Buss SJ, Messroghli D, Andre F, Lossnitzer D, Seitz S et al (2015) T1 mapping in dilated cardiomyopathy with cardiac magnetic resonance: quantification of diffuse myocardial fibrosis and comparison with endomyocardial biopsy. Eur Heart J Cardiovasc Imaging 16(2):210-216

24. Schulz-Menger J, Bluemke DA, Bremerich J, Flamm SD, Fogel MA, Friedrich MG et al (2013) Standardized image interpretation and post processing in cardiovascular magnetic resonance: Society for Cardiovascular Magnetic Resonance (SCMR) board of trustees task force on standardized post processing. J Cardiovasc Magn Reson 15:35

25. Cerqueira MD, Weissman NJ, Dilsizian V, Jacobs AK, Kaul S, Laskey WK et al (2002) Standardized myocardial segmentation and nomenclature for tomographic imaging of the heart. A statement for healthcare professionals from the Cardiac Imaging Committee of the Council on Clinical Cardiology of the American Heart Association. Circulation 105(4):539-542

26. Barbier CE, Bjerner T, Johansson L, Lind L, Ahlstrom H (2006) Myocardial scars more frequent than expected: magnetic resonance imaging detects potential risk group. J Am Coll Cardiol 48(4):765-771

27. Hosch W, Bock M, Libicher M, Ley S, Hegenbart U, Dengler TJ et al (2007) MR-relaxometry of myocardial tissue: significant elevation of $\mathrm{T} 1$ and $\mathrm{T} 2$ relaxation times in cardiac amyloidosis. Investig Radiol 42(9):636-642

28. Zhao L, Tian Z, Fang Q (2016) Diagnostic accuracy of cardiovascular magnetic resonance for patients with suspected cardiac amyloidosis: a systematic review and meta-analysis. BMC Cardiovasc Disord 16:129

29. Puntmann VO, Voigt T, Chen Z, Mayr M, Karim R, Rhode K et al (2013) Native T1 mapping in differentiation of normal myocardium from diffuse disease in hypertrophic and dilated cardiomyopathy. JACC Cardiovasc Imaging 6(4):475-484

30. McCrohon JA, Moon JC, Prasad SK, McKenna WJ, Lorenz $\mathrm{CH}$, Coats AJ et al (2003) Differentiation of heart failure related to dilated cardiomyopathy and coronary artery disease using 
gadolinium-enhanced cardiovascular magnetic resonance. Circulation 108(1):54-59

31. Yanagisawa F, Amano Y, Tachi M, Inui K, Asai K, Kumita S (2019) Non-contrast-enhanced T1 mapping of dilated cardiomyopathy: comparison between native T1 values and late Gadolinium enhancement. Magn Reson Med Sci 18(1):12-18

32. Mordi I, Carrick D, Bezerra H, Tzemos N (2016) T1 and T2 mapping for early diagnosis of dilated non-ischaemic cardiomyopathy in middle-aged patients and differentiation from normal physiological adaptation. Eur Heart J Cardiovasc Imaging 17(7):797-803

33. Kato S, Nakamori S, Bellm S, Jang J, Basha T, Maron M et al (2016) Myocardial native T1 time in patients with hypertrophic cardiomyopathy. Am J Cardiol 118(7):1057-1062

34. Dass S, Suttie JJ, Piechnik SK, Ferreira VM, Holloway CJ, Banerjee R et al (2012) Myocardial tissue characterization using magnetic resonance noncontrast $\mathrm{t} 1$ mapping in hypertrophic and dilated cardiomyopathy. Circ Cardiovasc Imaging 5(6):726-733

35. Abstracts of the 16th Annual SCMR (Society for Cardiovascular Magnetic Resonance) Scientific Sessions (2013) San Francisco, California, USA. January 31-February 3, 2013. J Cardiovasc Magn Reson 15 Suppl 1:E1-124, M1-14, O1-14, P1-300, T1-11, W-39

36. Shahbaz AU, Sun Y, Bhattacharya SK, Ahokas RA, Gerling IC, McGee JE et al (2010) Fibrosis in hypertensive heart disease: molecular pathways and cardioprotective strategies. J Hypertens 28(Suppl 1):S25-32

37. Hinojar R, Varma N, Child N, Goodman B, Jabbour A, Yu CY et al (2015) T1 mapping in discrimination of hypertrophic phenotypes: hypertensive heart disease and hypertrophic cardiomyopathy: findings from the international T1 multicenter cardiovascular magnetic resonance study. Circ Cardiovasc Imaging 8(12): 003285

38. Swoboda PP, McDiarmid AK, Erhayiem B, Broadbent DA, Dobson LE, Garg P et al (2016) Assessing myocardial extracellular volume by T1 mapping to distinguish hypertrophic cardiomyopathy from Athlete's heart. J Am Coll Cardiol 67(18):2189-2190

39. Nadjiri J, Zaschka AL, Straeter AS, Sauter A, Englmaier M, Weis F et al (2019) Evaluation of a shortened cardiac MRI protocol for left ventricular examinations: diagnostic performance of T1-mapping and myocardial function analysis. BMC Med Imaging 19(1):57

Publisher's Note Springer Nature remains neutral with regard to jurisdictional claims in published maps and institutional affiliations. 